\title{
REVUE
}

\section{L'INDUSTRIE LAITIÈRE A L'ÉTRANGER}

\author{
par \\ G. GÉNIN \\ Ingénieur E. P. C.
}

Dès le début de cette chronique, nous mentionnerons la publication, par la "Milk Industry Foundation" d'une petite brochure intitulée "Milk Facts " qui contient de très intéressants renseignements numériques relatifs à l'industrie mondiale des produits laitiers.

Avec une consommation par habitant et par an de 232 quarts de lait (un quart $=1$ litre 136) la Suisse est à la tête de tous les pays, viennent ensuite les Etats-Unis 153, le Danemark 144, la Tchéco-Slovaquie 136, les Pays-Bas 120, la Nouvelle-Zélande 112, la Grande-Bretagne, la France et l'Allemagne 92, l'Australie 88 , la Belgique 68 et l'Italie 28.

Le lait, sous une forme ou sous une autre, représente plus de $25 \%$ des 1.500 pounds d'aliments divers consommés chaque année par l'Américain moyen. Les Etats-Unis utilisent par an 48.777.000.000 quarts de lait, $29,2 \%$ de ce lait est consommé directement, $31,6 \%$ est utilisé par les laiteries pour la fabrication du beurre, 10,5\% par les fermiers pour la fabrication du beurre et $12,10 \%$ sont utilisés directement sur place par les fermiers. La fabrication du fromage consomme $5,9 \%$ de la totalité du lait produit, celle de la crème glacée $2,3 \%$ et celle du lait concentré $4,3 \%$.

\section{ALLEMAGNE}

Comment l'Allemagne a réorganisé la distribution du lait

On sait que beaucoup d'Etats se préoccupent actuellement de réorganiser leur industrie laitière, il est done intéressant de décrire rapidement ce qui a été fait en Allemagne depuis déjà quelques années en soulignant que les autorités chargées de cette réorganisation ont d'ailleurs tourné leurs efforts plus du côté distribution que du côté production.

Comme dans un grand nombre de pays d'Europe, les races de vache laitière et les méthodes d'élevage sont très diverses, suivant les régions de l'Allemagne. Dans le nord-ouest, ee sont les races de Frise et de Shorthorn qui prédominent et les fermiers s'attachent surtout à obtenir une production de lait très élevée. Dans l'Allemagne centrale, les races changent complètement de caractère et 
les animaux sont utilisés, non seulement comme producteurs de lait, mais également comme bêtes de somme.

Quand on étudie également comment est utilisé le lait en Allemagne, on est frappé par la proportion élevée de ce produit qui est consommé ou transformé sur place par les fermiers :

\begin{tabular}{|c|c|}
\hline Lait consommé et transformé par les fermiers & 39 \\
\hline Lait vendu pour la fabrication du beurre ............ & 38,8 \\
\hline Lait vendu pour d'autres fabrications $\ldots \ldots \ldots \ldots \ldots \ldots \ldots$ & 6,1 \\
\hline Lait vendu pour la consommation directe .. & 16,1 \\
\hline
\end{tabular}

La réorganisation a porté sur les points suivants : les agriculteurs allemands ont été invités plutôt que de continuer à fabriquer le beurre eux-mêmes, à le vendre à des laiteries mieux outillées pour produire un beurre de meilleure qualité et surtout pour assurer une meilleure utilisation des sous-produits, en particulier de la caséine et du petit-lait.

Les autorités se sont efforcées également de réduire les frais de traitement des produits laitiers et surtout les frais de distribution. Des ouvertures de crédit importantes ont été accordées aux laiteries pour leur permettre de réparer et surtout d'améliorer leur outillage. Par une réorganisation de la distribution on a pu réduire les frais de vente du lait et par conséquent, en maintenant les prix de vente au détail, augmenter la rétribution des producteurs. C'est ainsi que pour Hambourg, la différence entre le prix de vente au détail du lait et le prix payé au producteur, qui était de 0 mark 10 en 1932 a été abaissé à 0,075 R.-M. et que dans les villes moins peuplées cette marge est encore très sensiblement réduite.

Cette réorganisation de la production a eu également pour conséquence d'assurer une meilleure alimentation des grands centres. Actuellement, par exemple, l'alimentation en lait de la ville de Berlin est assurée par des producteurs situés au maximumà 150 kilomètres de la ville alors qu'auparavant certains producteurs se trouvaient à 300 kilomètres, d'où frais considérables de transport et diminution de la qualité du lait.

Les autorités attachent une grande importance à la qualité du lait, des standards de qualité ont été établis et des primes sont accordées aux producteurs qui fournissent, grâce aux conseils des techniciens, un lait répondant à ces standards.

Enfin, les vendeurs de lait au détail doivent désormais posséder une licence et, par une réduction du nombre de ces licences, on a accru le chiffre d'affaires de chaque établissement et réduit en proportion ses frais généraux. En 1932, il existait à Berlin 6.200 
laiteries de détail vendant en moyenne 70 litres de lait par jour, ce chiffre a été réduit à $\mathbf{5 . 9 7 5}$ établissements qui distribuent chacun 110 litres par jour en moyenne.

\section{L'application du procédé Hofius}

On sait que le procédé Hofius, d'apparition relativement récente permet de conserver les produits laitiers et en particulier le lait à l'état frais, en le plaçant dans des récipients contenant de l'oxygène sous pression et maintenus à une basse température (au-dessous de $10^{\circ}$ C.).

On envisage d'utiliser ce procédé dans toutes les régions non productrices de lait, où il n'est pas possible d'obtenir du lait frais, ainsi qu'à bord des paquebots et à l'occasion d'expéditions militaires. La station expérimentale de Kiel, chargée de l'étude de ce procédé, indique que son emploi est conditionné par le prix des récipients résistant à la pression.

\section{L'enregistrement des vaches laitières}

Les autorités allemandes ont décidé d'étendre à la totalité des régions de l'Allemagne, la décision prise, il y a quelques années, de tenir un registre des vaches laitières sur lequel figure également la production de lait de ces animaux. En 1937, 55\% des vaches étaient déjà enregistrées ; actuellement cette proportion s'est élevée à $95 \%$.

C'est pour répondre au désir du gouvernement de voir le pays se suffire à lui-même, que cette décision qui permet d'assurer un meilleur contrôle de l'Etat a été prise et, au cours de ces dernières années, l'Allemagne a produit $85 \%$ du beurre qui lui est nécessaire et $90 \%$ du fromage. Ce contrôle va permettre également de choisir pour l'élevage, les races les plus productrices et de déterminer les conditions de vie et d'alimentation des animaux qui sont favorables à une forte production de lait. Les experts s'élèvent contre l'opinion suivant laquelle une forte production de lait est obtenue aux dépens de la santé des animaux. Ils affirment qu'une production-annuelle de plus de 9.000 litres peut être obtenue sans diminuer la fertilité des vaches au point de vue reproduction et sans altérer leur santé.

\section{ANGLETERRE}

\section{La création d'un bureau impérial pour l'étude des questions laitières}

En 1936, la British Commonwealth Conference, étudiant les activités des organisations contrôlées par le Conseil exécutif des Bureaux Impériaux d'agriculture, avait émis un vœu en faveur de 
la création d'un bureau Impérial de la Science laitière, qui aurait pu être installé à l'Institut national de recherches de laiterie de Reading.

En réponse à ce vœu, ee bureau vient d'être constitué et son siège se trouve à Shinfield, près de Reading. La présidence en a été remise au Professeur H. D. KAY, directeur de l'Institut de Reading et les dépenses de cette nouvelle organisation seront couvertes par des subventions des divers gouvernements de l'Empire britannique et des Bureaux d'Agriculture.

Le rôle de ce bureau est de réunir et d'analyser les travaux qui eoncernent l'industrie laitière publiés dans le monde entier, de réunir toutes informations économiques ou autres relatives à cette industrie et de les faire connaître aux intéressés de l'Empire, soit dans des publications, soit sous forme de communications privées. Le Bureau aura également comme rôle d'établir et de maintenir des contacts entre les chercheurs britanniques et de faciliter par tous les moyens les échanges d'idées.

\section{L'aluminium à la foire des industries britanniques}

A la dernière foire des industries britanniques, l'intérêt des visiteurs et en particulier des représentants de l'industrie laitière a été attiré par le stand de la British. Aluminium Co Ltd, le plus important producteur d'aluminium métal des Iles britanniques.

Parmi les nouveautés présentées à ce stand, signalons les principaux alliages d'aluminium caractérisés par la facilité de leur usinage et leur résistance à la corrosion, le procédé de traitement superficiel Brytal employé pour augmenter le pouvoir réfléchissant de la lumière des réflecteurs en aluminium, le procédé anodique qui, appliqué à l'aluminium et à certains de ses alliages, améliore l'aspect de ces métaux et augmente leur résistance au ternissement, de telle sorte que le nettoyage de leur surface exige un simple lavage à l'eau savonneuse.

Une place importante était également réservée à l'aluminium comme feuille d'emballage, l'emploi de ces feuilles permettant de protéger les produits emballés contre l'action de la lumière, de la chaleur et des agents atmosphériques.

\section{La diminution de la production anglaise de beurre et de fromage}

La production de fromage en Angleterre et au Pays de Galles a été, au cours de l'année ayant pris fin le 30 septembre 1938, la plus faible qu'on ait enregistrée depuis que des statistiques de cette production sont établies. Il en a été de même pour la production du beurre, ainsi que le montre le tableau ci-après. 
Année

1933-1934...............

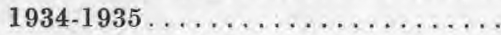

$1935-1936 \ldots \ldots \ldots \ldots \ldots \ldots \ldots$

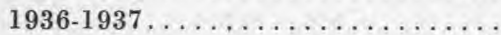

$1937.1938 \ldots \ldots \ldots \ldots \ldots \ldots \ldots \ldots$

$\begin{array}{cr}\text { Beurre Cwt } & \text { Fromage Cwt } \\ 630.000 & 840.000 \\ 730.000 & 1.040 .000 \\ 820.000 & 970.000 \\ 750.000 & 730.000 \\ 640.000 & 700.000\end{array}$

Il a été utilisé environ 20 millions de gallons de lait en 1937-1938 pour la fabrication du fromage dans les fermes et 58 millions dans les fromageries. On constate d'ailleurs, depuis quelques années, une plus grande tendance de la part des fermiers à vendre leur lait plutôt que de l'utiliser pour la préparation du fromage (surtout du Cheshire).

Rappelons que la Grande-Bretagne ne produit que $10 \%$ du beurre nécessaire à sa consommation et $30 \%$ du fromage.

\section{SUÈDE}

\section{La publicité en faveur de la consommation du lait}

Le lait et les produits laitiers occupaient une très large place dans une exposition peu fréquente, qui a été organisée récemment à Stockholm en vue d'éduquer le public et de lui faire connaître la valeur nutritive des divers aliments qu'il a à sa disposition.

Les visiteurs étaient tout d'abord instruits sur la question de la conservation du lait. On présentait les meilleurs types de pots dans lesquels on peut conserver le lait, les meilleures méthodes pour maintenir le lait frais et les procédés convenables et efficaces pour nettoyer les récipients ayant contenu du lait.

Le beurre était présenté comme la matière grasse ayant la plus grande valeur alimentaire et on comparait en particulier sa teneur en vitamines à celle de la margarine et des autres graisses. Le fromage était signalé à l'attention du public comme une des formes les plus économiques des produits nutritifs.

L'importance du lait dans l'obtention d'une bonne dentition: était indiquée, en même temps qu'on rappelait que la consommation régulière de lait permet aux femmes d'acquérir une ligne svelte.

Le succès de cette exposition a été tel qu'on envisage son transport dans les villes les plus importantes de la Suède.

\section{ÉTATS-UNIS}

\section{L'enrichissement du lait en vitamine D par le procédé A.R.P.I.}

Parmi les méthodes d'enrichissement du lait en vitamine $D$, les plus courantes sont l'irradiation et l'addition de concentrés de vitamine $\mathrm{D}$ soit à la nourriture des animaux, soit directement au 
lait. Le nouveau procédé A. R. P. I. permet d'ajouter le concentré au lait condensé.

Ce concentré est constitué par une solution d'ergostérol activé dans de l'huile de beurre, on l'ajoute au lait condensé, on introduit le mélange dans des boites et on stérilise. Ce procédé a été homologué par le Conseil des produits alimentaires de l'Association médicale américaine.

Aucun appareil particulier n'est nécessaire pour préparer ce lait enrichi en vitamine $\mathrm{D}$; il suffit de verser le concentré directement dans le lait eoncentré avant la pasteurisation et, comme ce concentré est déjà homogénéisé, il se distribue uniformément dans le lait sous la seule influence de l'agitation qui se produit au cours de la pasteurisation.

\section{Nouvel appareil à arc de carbone pour l'irridiation du lait}

Une firme américaine vient de présenter un nouvel appareil pour l'irradiation du lait, qui se caractérise par son rendement élevé, sa facilité d'entretien et de nettoyage et sa faible dépense d'énergie. Il est constitué, essentiellement, par un cylindre en acier inoxydable, portant à sa partie supérieure un cône centré dans l'appareil, sur lequel l'on déverse le lait de telle sorte que celui-ci s'écoule dans l'appareil sous forme d'un voile cylindrique très mince. Une lampe à arc de carbone, alimentée par du courant triphasé est placée au centre de l'appareil et émet une lumière ultra-violette intense qui irradie le lait pendant son court passage dans l'appareil.

Des dispositifs permettent de régulariser et d'uniformiser le passage du lait et l'appareil peut être réglé pour l'irradiation du lait normal ou du lait concentré. Tous les éléments de l'appareil placés au contact du lait sont en acier inoxydable et soudés. Le débit peut être réglé entre 2.000 à 8.000 kilogrammes de lait à l'heure.

\section{Le manque de vitamine $A$ dans la nourriture diminue l'acuité visuelle}

Des savants du Bureau of Home Economics du Ministère de l'Agriculture des Etats-Unis : L. A. BоонеR et E. Collison, ont observé que le manque de vitamine A dans la nourriture diminue les facultés visuelles et peut être la cause de graves accidents d'automobiles, ear, dans ce cas, le conducteur déficient ne retrouve que très lentement ses facultés lorsqu'il a été ébloui par les phares d'une voiture venant à sa rencontre. La vision latérale est également réduite et cela gêne alors la marche des piétons sur une route, On peut parer à cet inconvénient en ajoutant à la nourriture habituelle des substances riches en vitamine A, parmi lesquelles il faut citer le lait, la crème, les œufs, les fromages, les tomates et les légumes verts. 